\title{
Adolescent Motherhood and HIV in South Africa: Examining Prevalence of Common Mental Disorder
}

\author{
Kathryn J. Roberts ${ }^{1}\left(\mathbb{0} \cdot\right.$ Colette Smith $^{1} \cdot{\text { Lucie } \text { Cluver }^{2,3} \cdot \text { Elona Toska }^{2,4,5} \cdot \text { Siyanai Zhou }}^{4} \cdot$ Mark Boyes $^{6}$. \\ Lorraine Sherr ${ }^{1}$
}

Accepted: 16 September 2021 / Published online: 27 September 2021

(c) The Author(s) 2021

\begin{abstract}
The mental health of adolescents (10-19 years) remains an overlooked global health issue, particularly within the context of syndemic conditions such as HIV and pregnancy. Rates of pregnancy and HIV among adolescents within South Africa are some of the highest in the world. Experiencing pregnancy and living with HIV during adolescence have both been found to be associated with poor mental health within separate explorations. Yet, examinations of mental health among adolescents living with HIV who have experienced pregnancy/parenthood remain absent from the literature. As such, there exists no evidence-based policy or programming relating to mental health for this group. These analyses aim to identify the prevalence of probable common mental disorder among adolescent mothers and, among adolescents experiencing the syndemic of motherhood and HIV. Analyses utilise data from interviews undertaken with 723 female adolescents drawn from a prospective longitudinal cohort study of adolescents living with HIV $(n=1059)$ and a comparison group of adolescents without HIV ( $n=467)$ undertaken within the Eastern Cape Province, South Africa. Detailed study questionnaires included validated and study specific measures relating to HIV, adolescent motherhood, and mental health. Four self-reported measures of mental health (depressive, anxiety, posttraumatic stress, and suicidality symptomology) were used to explore the concept of likely common mental disorder and mental health comorbidities (experiencing two or more common mental disorders concurrently). Chi-square tests (Fisher's exact test, where appropriate) and Kruskal Wallis tests were used to assess differences in sample characteristics (inclusive of mental health status) according to HIV status and motherhood status. Logistic regression models were used to explore the cross-sectional associations between combined motherhood and HIV status and, likely common mental disorder/mental health comorbidities. $70.5 \%$ of participants were living with HIV and $15.2 \%$ were mothers. $8.4 \%$ were mothers living with HIV. A tenth (10.9\%) of the sample were classified as reporting a probable common mental disorder and $2.8 \%$ as experiencing likely mental health comorbidities. Three core findings emerge: (1) poor mental health was elevated among adolescent mothers compared to never pregnant adolescents (measures of likely common mental disorder, mental health comorbidities, depressive, anxiety and suicidality symptoms), (2) prevalence of probable common mental disorder was highest among mothers living with HIV (23.0\%) compared to other groups (Range:8.5-12.8\%; $\left.\mathrm{X}^{2}=12.54, \mathrm{p}=0.006\right)$ and, (3) prevalence of probable mental health comorbidities was higher among mothers, regardless of HIV status (HIV \& motherhood $=8.2 \%$, No HIV \& motherhood $=8.2 \%, \mathrm{X}^{2}=14.5, \mathrm{p}=0.002$ ). Results identify higher mental health burden among adolescent mothers compared to never-pregnant adolescents, an increased prevalence of mental health burden among adolescent mothers living with HIV compared to other groups, and an elevated prevalence of mental health comorbidities among adolescent mothers irrespective of HIV status. These findings address a critical evidence gap, highlighting the commonality of mental health burden within the context of adolescent motherhood and HIV within South Africa as well as the urgent need for support and further research to ensure effective evidence-based programming is made available for this group. Existing antenatal, postnatal, and HIV care may provide an opportunity for mental health screening, monitoring, and referral.
\end{abstract}

Keywords Common mental disorder $\cdot$ Mental health $\cdot$ Adolescent motherhood $\cdot$ HIV $\cdot$ South Africa $\cdot$ Sub-Saharan Africa

Extended author information available on the last page of the article 


\section{Introduction}

Sub-Saharan Africa is home to the fastest growing adolescent population (10-19 years) [1] in the world, expected to reach 435 million by 2050 [2]. As such, the promotion of adolescent wellbeing - of which mental health is a core component-is critical to the success and prosperity of individuals, families, and to the region as a whole. Poor mental health within adolescence has been found to impact both physical and mental morbidity within adulthood [3-9] and, has been found to have broad societal level impacts on healthcare systems, workforce engagement and, consequently, broader socio-economic outcomes [10-12]. Despite this, mental health is an often neglected global health priority, particularly with regard to children and adolescents [9, 13-15]. Yet, 50\% of mental health disorders are established before 14 years of age, and $75 \%$, before the age of 24 years [3]. Globally, mental health disorders impact approximately 10-20\% of children and adolescents $[13,16]$ however, there remains a dearth of prevalence estimates from low and middle income countries which may skew such global data [17-20]. Likewise, there is limited data relating to the prevention and treatment of poor mental health among children and adolescence outside of the field of developmental disability [20]. To promote the success and prosperity of adolescents within the sub-Saharan African region, an increased understanding of mental health, inclusive of when poor mental health may be compounded by other syndemic conditions (in this instance adolescent pregnancy and HIV), is necessary to inform impactful policy and programming relating to the assessment, treatment, and overall experience of mental health for adolescents.

South Africa has one of the highest rates of adolescent pregnancy (10-19 years) in the world-recent estimates suggest that $19 \%$ (95\% confidence interval: 16-22\%) of female adolescents have experienced pregnancy [21]. In addition to navigating a broad development period, denoted by substantial psychological, social and, biological changes, pregnant and parenting adolescents (both adolescent mothers and fathers) must also traverse pregnancy, parenting, and childrearing. Adolescent pregnancy and parenting are associated with adverse outcomes including a higher likelihood of complications during pregnancy and childbirth, adverse neonatal outcomes, repeat pregnancy, harsher parenting practices and development difficulties among their children $[22,23]$. In addition, adolescent pregnancy has previously been found to be associated with poor mental health [23, 24]. Given the sensitivity of the adolescent developmental period, poor mental health during this phase may present distinctive challenges compared to pregnancy and parenthood during adulthood.
Poor mental health within pregnancy and parenthood may have negative implications for maternal and child health, attachment and bonding, child development outcomes [24-28] and, at its worst, mortality — with maternal suicide linked to poor mental health. Yet, there is limited literature exploring adolescent mental health within pregnancy and parenting from the sub-Saharan African region. Here, adolescents are exposed to numerous risk factors for poor mental health including poverty, violence exposure and, high levels of communicable disease (i.e. HIV). Risk of poor mental health may therefore be further compounded by the experience of adolescent pregnancy within such settings.

The HIV epidemic within South Africa remains the largest in the world despite huge advances within the past decades. Approximately a fifth of the population $(15+$ years $)$ are living with HIV [29]. Among adolescents, HIV has been found to be associated with poor mental health. A recent systematic review identified a high prevalence of mental health problems among adolescents living with HIV (ALHIV), with $24-27 \%$ of adolescents identified as experiencing psychiatric disorder and 30-50\% showing behavioural or emotional difficulties [30]. Yet, the capacity for mental health treatment and care remains restricted across sub-Saharan Africa. For adolescents living with HIV, poor mental health has implications for treatment adherence [31,32] which in turn has implications for health outcomes and onward HIV transmission.

Given that both pregnancy and living with HIV have been found to be independently associated with poor mental health among adolescents [23, 33, 34], experiencing the syndemic of both adolescent pregnancy and living with HIV may compound poor mental health experience. Such experiences may also have a bidirectional relationship with poor mental health [23, 33, 34]. Poor mental health has previously been found to be prevalent among adult populations living with HIV within pregnancy and the postpartum period [35] - however, explorations of mental health among adolescent populations are yet to be undertaken. For those adolescents living with HIV experiencing pregnancy/parenthood, there remains additional considerations such as health, stigma, medication adherence and, both postnatal and perinatal HIV transmission [36] —all of which may be impacted by mental health experience. Thus, it is important to develop an understanding of mental health within the context of adolescent parenthood and HIV to establish the impacts for both adolescents and their children.

The mental health of adolescents living with HIV who have experienced pregnancy/parenthood (both mothers and fathers) in sub-Saharan Africa and, as such, South Africa, is a neglected topic [37]. A recent systematic review exploring common mental disorder within the context of adolescent pregnancy and HIV in sub-Saharan Africa [37] identified 
only a single prevalence study (undertaken in Kenya) relating to depressive symptomology among a sub-sample of adolescents living with HIV who were currently pregnant $(n=14)$ [28]. Given the small sample size as well as the sole focus on depressive symptomology and the pregnancy period within this study [38], an understanding of common mental disorder among adolescents living with HIV who are experiencing/have experienced pregnancy remains lacking. The review identified no studies relating to risk and protective factors for mental health, and no studies relating to the interventions for poor mental health among this group [37]. These adolescents have seemingly been side-lined from mental health agendas - there exists no evidence-based policy or programming available for this group. Explicit examinations of the mental health experience of adolescents living with HIV who have experienced pregnancy/parenthood are yet to be undertaken. To build an evidence base, the first step is to establish an understanding of potential mental health need, and how this need relates to adolescent pregnancy and/ or HIV status.

This study aims to determine the prevalence of likely common mental disorder among adolescents, and specifically among those who have experienced motherhood and are living with HIV in South Africa. In line with the definition of common mental disorder utilised within the recent systematic review exploring this topic area [37], likely experience of common mental disorder was defined as the presence of at least one of depressive and anxiety, posttraumatic stress, and suicidality symptomology. Globally, depression and anxiety are prominent causes of illness and disability among adolescents $[39,40]$. Given the commonality of trauma experience among South African adolescents [41], the severity of suicidality symptomology (i.e. mortality) [42] and, the potential comorbidity of posttraumatic stress and suicidality with depression and general anxiety, a broad definition of likely common mental disorder (depressive, anxiety, posttraumatic stress, and suicidality symptomology) was utilised within analyses to identify need and where support for adolescents may be required.

\section{Methods}

\section{Participants and Procedure}

Data utilised within these analyses are drawn from a large prospective longitudinal cohort study of adolescents in the Eastern Cape province of South Africa $(n=1526)$. One thousand and fifty-nine adolescents living with HIV were recruited to the study utilising records from 53 public health facilities providing antiretroviral therapy to adolescents within the province. Sampling was undertaken in two stages: (1) public health facilities were identified through the national Department of Health register and, (2) all adolescents on public health facility records that had initiated treatment in the previous 3 years were approached inclusive of those disengaged from care. Adolescents were followed up utilising community tracing methods to ensure the inclusion of both those engaged and disengaged with HIV services. At baseline, $90.1 \%$ of the eligible sample identified through clinical records were interviewed. The comparison group $(n=467)$ were age-matched, and selected from the same environments, co-residing with or near adolescents living with HIV study participants also completed interviews. These participants self-reported that they were not living with HIV and had not initiated antiretroviral therapy and had not experienced possible opportunistic infections, nor had history of familial HIV/AIDS, thus classified as not living with HIV for the purpose of these analyses. Baseline data collection was undertaken between February 2014 and September 2015. Follow-up data was collected between November 2015 and February 2017. The cohort had a 95.3\% retention rate at follow-up $(n=1454)$.

All adolescents and caregivers (if adolescents were $<18$ years of age) provided informed consent. To protect participants confidentially interviews were conducted in a private space chosen by the participant. Given the risk of stigma relating to HIV within the communities from which data were gathered, to further protect confidentiality of participants the study was presented as a broad study focusing on youth access to health and social care services. All participants completed interviews consisting of a detailed study questionnaire consisting of validated scales and study specific questions focused on sociodemographic characteristics, health (inclusive of mental health screening measures), relationships, communities, schooling, and managements of HIV (if appropriate). Study questionnaires were developed in conjunction with adolescents (pre-piloted with a teen advisory group local to South Africa prior to data collection), as well as local, national, regional, and global organisations. Participants completed questionnaires in their language of choice (isiXhosa or English), and data was translated and back translated if appropriate.

Analyses present data relating to adolescent motherhood (previous pregnancy between 10 and 19 years of age). Only data for female adolescents' $\leq 20$ years of age were included within these analyses. Females who were 20 years of age were retained in the sample if they were within 40 weeks (average length of a full-term pregnancy) of their twentieth birthday to ensure that any reporting of pregnancy was between 10 and 19 years of age. Dates of birth and dates of interview were used to ascertain eligibility. Adolescent motherhood status was ascertained from participant selfreport. Given the potentially differing mental health profiles of current adolescent mother and adolescents who had experienced elective abortion, miscarriage or still birth, 
Fig. 1 Participant selection criteria

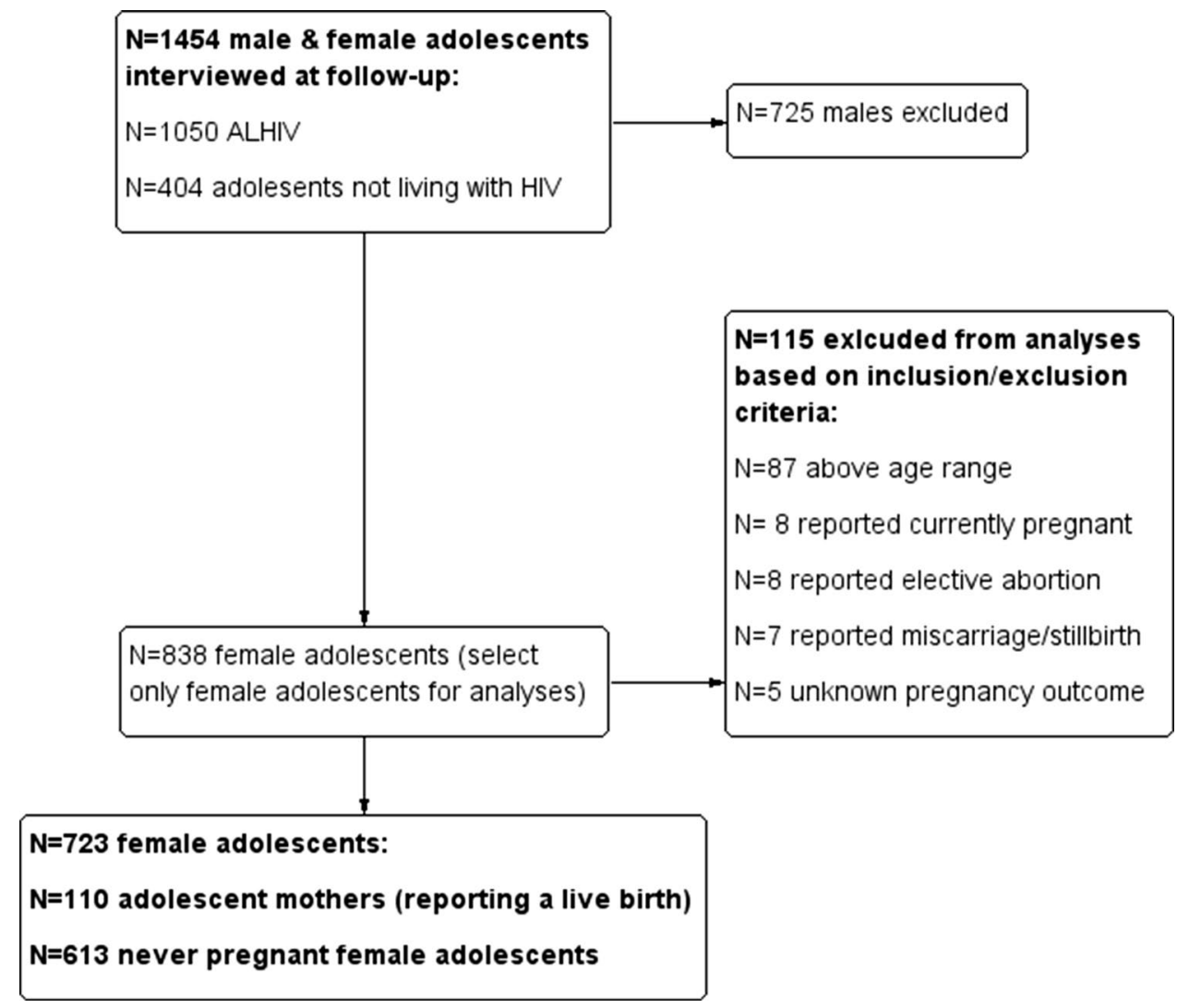

adolescents who reported a pregnancy but did not report a live birth were excluded from these analyses. In total, 723 adolescents were included within analyses (see Fig. 1).

\section{Measures}

These analyses utilise cross-sectional data. Both baseline and follow-up data were used to corroborate reported adolescent pregnancy within the sample, all other measures were obtained within the follow-up round of data collection (2015-2017; total sample $n=1454$; see Fig. 1).

\section{Sociodemographic Characteristics}

Sociodemographic characteristics were routinely gathered during baseline data collection and corroborated during follow-up data collection. Sociodemographic characteristics included age, biological sex, dwelling location, housing, school enrolment, orphanhood status and cash grant receipt via self-report measures.

\section{Motherhood Status}

Adolescent motherhood (previous pregnancy and live birth between 10 and 19 years) was identified using two selfreport measures [having had a previous pregnancy resulting in a live birth and having one or more biological child(ren)]. Adolescents were classified as experiencing motherhood if they scored on any one of the measures of motherhood (above). Adolescents who had experienced miscarriage, abortion, stillbirth, or the outcome of the pregnancy was unknown were excluded from analyses (see Fig. 1). Whether the pregnancy was planned and/or wanted (most recent pregnancy), age of last pregnancy (years), the number of previous pregnancies and the number of children were obtained from participant self-report.

\section{HIV Status}

HIV status was ascertained through clinical notes and corroborated by participant or caregiver self-report on a caseby-case basis.

\section{Mental Health Status}

All participants within the sample responded to all items of the four validated mental health scales used within these analyses. Cut-off scores were utilised to ascertain prevalence estimates of probable disorder.

Overall Mental Health Status Two measures of overall mental health status are presented within analyses; (1) Any com- 
mon mental disorder (CMD); If a participant scored above the cut-off on any of the mental health measures within the study (see below) they were classified as experiencing poor mental health. (2) Any mental health comorbidities (MHCs); If a participant scored above the cut-off on two or more of the mental health measures within the study (see below) they were classified as having mental health comorbidities.

Depressive Symptomology Depressive symptomology was measured using the 10-item Child Depression Inventory short form (CDI-S) [43, 44]. Items consisted of a series of statements (i.e. 'I like myself', 'I don't like myself' 'I hate myself') for which adolescents had to choose the statement that reflected how they felt. Items were scored $0-2$ $(0=$ absent symptoms, $1=$ mild symptoms and, $2=$ defini tive symptoms) [43, 44]. Scores of $\geq 3$ (based on mild/ definitive symptoms due to the small number of participants reporting definitive symptoms) [45] were used to indicate symptomology consistent with a positive screen for probable depression (binary; yes/no). The CDI-S been previously found to be highly correlated $(r=0.89)$ with the broader 27-item CDI scale as such scores were prorated based on the suggested inclusive cut-off within the within the full scale $[43,46,47]$. A cut-off $\geq 3$ has been previously been used in high income contexts [48]. The CDI (from which the CDI-S is derived) has generally been found to have strong psychometric properties and is well validated in the sub-Saharan African region (CDI-S: $\alpha=0.66$ in the current sample) [4952]. The CDI-S has previously been used with adolescent populations in South Africa. $n$ adolescents [47, 53-56].

Anxiety Symptomology Anxiety symptomology was measured using an abbreviated version (14 items) of the Children's Manifest Anxiety Scale-Revised (RCMAS) [57, 58]. Adolescents identified if statements (i.e. "I worry a lot of the time") were true for them. The 14 items were scored as "yes" (1) or "no" (0); indicative of experience consistent with anxiety symptomology and not, respectively. Scores $\geq 10$ were used to indicate symptomology consistent with a positive screen for anxiety [57, 58]. The RCMAS has been validated within sub-Sharan Africa and shows good internal consistency among HIV-affected children and adolescents ( $\alpha=0.85$ in the sample) [59].

Posttraumatic Stress Symptomology Posttraumatic stress symptomology was measured using a 19-item version of the Child PTSD checklist [60, 61]. Adolescents were asked to think about something upsetting or frightening that has happened in their lives and identify how often they felt a series of items (i.e. "Do you get upset when you think about what happened?" Items were scored 0-3 (0 "not at all", 1 "some of the time", 2 "most of the time", 3 "all of the time").
Items within the checklist represent four domains relating to posttraumatic stress disorder (re-experience, avoidance, hyperarousal and, dysphoria), based on a four factor model proposed by Boyes et al. [61] for South African Adolescents [60]. Participants were classified as experiencing symptomology consistent with a probable positive screen for posttraumatic stress disorder if they scored on items across all four domains with affirmative responses (i.e. "most of the time"/"all of the time") in the following frequencies: reexperience $>=1$, avoidance $>=1$, hyperarousal $>=2$, dysphoria $>=2$ [61]. Classifications were used to determine the presence of posttraumatic stress based on the DSM-5 criteria and were prorated based on the full Child PTSD scale $[60,62,63]$. The Child PTSD checklist showed good internal consistency within the sample $(\alpha=0.84)$, has been widely used among adolescents and youth with South Africa $[64,65]$ and, the 19-item scale has been validated within the South African context [61].

Suicidality/Self Harm Symptomology Suicidality/selfharm was measured using the five-item Mini International Neuropsychiatric Interview (MINI-KID; scored 0-5) [66]. The MINI-KID used the following questions to identify suicidal symptoms: "In the past month did you: wish you were dead?" "Want to hurt yourself?" "Think about killing yourself?" "Think of a way to kill yourself?" "Attempt suicide?" Participants responded "yes" (1) or "no" (0). Participants were classified as reporting suicidal symptoms if they scored on any item on the MINI-Kid [66]. Globally, the MINI-KID has been extensively validated, demonstrates good internal consistency ( $\alpha=0.89$ in the current sample), and good test-retest reliability [66-68].

\section{Statistical Analyses}

All analyses were undertaken using STATA v.15. [69] Chisquare tests (Fisher's exact test, where appropriate) and Kruskal Wallis tests were used to explore sample characteristics (inclusive of mental health status) according to motherhood status. Prevalence and associations of likely common mental disorder with adolescent motherhood, HIV status (including the two factors combined) were described descriptively and assessed using chi-square tests. Finally, logistic regression models were used to explore the crosssectional associations between motherhood and HIV status (inclusive of interaction effects) and common mental disorder. Interaction effects of motherhood and maternal HIV status were assessed by introducing interaction terms into the multivariable models. Adjusted odds ratios from the models including interaction terms were used to develop forest plots as a visual representation of the odds of experiencing common mental disorder among adolescent mothers who are living with HIV. Confounding factors were included 
in multivariable regression models if they were identified as being relevant factors within the literature of interest or found to be associated $(p=<0.2)[70,71]$ with either, or both, the predictor and outcome variables.

\section{Results}

\section{Sociodemographic Characteristics}

Table 1 presents sample characteristics stratified according to motherhood status. The prevalence of adolescent motherhood in the sample was $15.2 \%$. The median age of the sample was 15 years (IQR: 13-18 years). Adolescent mothers within the sample were older compared to never pregnant adolescents [19 (IQR: 18-20) vs. 15 (IQR: 13-17) years, $\left.\mathrm{X}^{2}=166.9, \mathrm{p}=0.0001\right]$. Over a quarter $(27.1 \%)$ resided in a rural area and $15.4 \%$ lived in informal (shack) housing. Adolescent mothers were more likely to be living in informal housing comparative to never pregnant adolescents ( $23.5 \%$ vs. $\left.14.1 \%, \mathrm{X}^{2}=5.73, \mathrm{p}=0.02\right)$. Over half of participants were orphans $(52.7 \%)$. On average, the highest school grade passed was grade 9 [IQR: 7-11] and, $6.4 \%$ were not in receipt of social protection in the form of cash grants. The majority of the sample were living with HIV (70.5\%). Adolescent mothers were less likely to be living with HIV compared to never pregnant adolescents within the sample (55.5\% vs. $\left.73.3 \%, \mathrm{X}^{2}=14.21, \mathrm{p}=<0.0001\right)$. See Table 1 .

\section{Characteristics of Adolescent Motherhood}

Eight respondents reported giving birth to more than one child (1.3\%). The average reported age of last pregnancy within the full sample was 17 years (IQR: 16-18; Range 14-20 years). A fifth (20.9\%) of adolescents reporting pregnancy reported that their last pregnancy was before the age of 16 years. Adolescents living with HIV reported a higher age at last pregnancy compared to those who were not living with HIV [17 (IQR:15-19) years vs. 17 (IQR: 15-18) years, $\left.X^{2}=6.69, p=0.009\right]$. Almost all pregnancies (most recent) were unplanned or unwanted among adolescents- $98.8 \%$ of pregnancies were unplanned and, $98.8 \%$ of pregnancies were unwanted.

\section{Prevalence of Common Mental Disorder}

Table 2 presents the prevalence of probable common mental disorder for the total sample and according to the experience of adolescent motherhood. Eighty-five participants $(10.9 \%)$ scored above the predetermined cut-off on at least one screening measure for mental health (depression, anxiety, posttraumatic stress, suicidality) and were classified as experiencing a probable common mental disorder. Prevalence of probable mental health comorbidities (scoring above the predetermined two or more measures for different mental health) was $2.8 \%$ in the sample. Within individual scales, $6.9 \%$ of the sample were classified as experiencing depressive symptomology, $1.4 \%$ were classified as experiencing anxiety symptomology, $0.6 \%$ reported posttraumatic stress symptomology and, $6.1 \%$ reported suicidality symptomology. Adolescent mothers reported a greater prevalence of probable common mental disor$\operatorname{der}\left(18.2 \%\right.$ vs. $\left.9.6 \%, \mathrm{X}^{2}=7.02, \mathrm{p}=0.008\right)$ and probable mental health comorbidities $\left(8.2 \%\right.$ vs. $1.8 \%, \mathrm{X}^{2}=14.15$, $\mathrm{p}=<0.0001)$ compared to adolescents who had never been pregnant. Within individual scales, adolescent mothers reported a greater prevalence of depressive, anxiety, and suicidality symptoms (see Table 2) than adolescents who had not experienced a pregnancy.

Table 1 Sample characteristics

\begin{tabular}{|c|c|c|c|c|}
\hline & \multicolumn{3}{|l|}{$\mathrm{N}(\%) / \mathrm{M}(\mathrm{IQR})$} & \multirow[t]{2}{*}{$\mathrm{X}^{2}, \mathrm{p}$ value } \\
\hline & Total sample $(\mathrm{n}=723)$ & $\begin{array}{l}\text { Adolescent } \\
\text { mother } \\
(\mathrm{n}=110)\end{array}$ & $\begin{array}{l}\text { Never preg- } \\
\text { nant adolescent } \\
(\mathrm{n}=613)\end{array}$ & \\
\hline \multicolumn{5}{|c|}{ Sociodemographic characteristics } \\
\hline Current age (years) & $15(13-18)$ & $19(18-20)$ & $15(13-17)$ & $166.86,0.0001$ \\
\hline Dwelling location (rural)* & $195(27.1 \%)$ & $37(33.6 \%)$ & $158(25.9 \%)$ & $2.86,0.09$ \\
\hline Housing (informal)* & $109(15.4 \%)$ & $23(23.5 \%)$ & $86(14.1 \%)$ & $5.73,0.02$ \\
\hline $\begin{array}{l}\text { Orphanhood status (one or } \\
\text { both parents have died) }\end{array}$ & $381(52.7 \%)$ & $52(47.3 \%)$ & $329(53.7 \%)$ & $1.53,0.22$ \\
\hline Enrolled in school & $628(86.9 \%)$ & $58(52.7 \%)$ & $570(93.0 \%)$ & $132.44,0.0001$ \\
\hline Cash grant receipt & $677(93.6 \%)$ & $103(93.6 \%)$ & $574(93.6 \%)$ & $0.00,1.00$ \\
\hline Living with HIV & $510(70.5 \%)$ & $61(55.5 \%)$ & $449(73.3 \%)$ & $14.21,<0.0001$ \\
\hline
\end{tabular}

Bold indicates statistical significance $(p<0.05)$

*Missing data (total $\mathrm{n}$ included in analyses): Dwelling location $(\mathrm{n}=721) \mid$ Housing $(\mathrm{n}=709)$ 
Table 2 Mental health outcomes stratified according to adolescent motherhood

\begin{tabular}{|c|c|c|c|c|}
\hline \multirow[t]{2}{*}{ Mental health outcomes } & \multicolumn{3}{|l|}{$\mathrm{N}(\%)$} & \multirow[t]{2}{*}{$\mathrm{X}^{2}, \mathrm{p}$ value } \\
\hline & $\begin{array}{l}\text { Total sample } \\
(\mathrm{n}=723)(\%)\end{array}$ & $\begin{array}{l}\text { Adolescent mother } \\
(\mathrm{n}=110)(\%)\end{array}$ & $\begin{array}{l}\text { Never pregnant adoles- } \\
\text { cent }(\mathrm{n}=613)(\%)\end{array}$ & \\
\hline Any common mental disorder & $79(10.9)$ & $20(18.2)$ & $59(9.6)$ & $7.02,0.008$ \\
\hline Any mental health comorbidities & $20(2.8)$ & $9(8.2)$ & $11(1.8)$ & $14.15,<0.0001$ \\
\hline Depressive symptoms [scoring above cut-off $(\geq 3)$ ] & $50(6.9)$ & $14(12.7)$ & $36(5.9)$ & $6.80,0.009$ \\
\hline Anxiety symptoms (scoring above cut-off $\geq 10$ ) & $10(1.4)$ & $4(3.6)$ & $6(1.0)$ & $4.83,0.05$ \\
\hline Posttraumatic stress symptoms (scoring above cut-off) & $4(0.6)$ & $0(0.0)$ & $4(0.7)$ & $0.72,1.00$ \\
\hline Suicidality symptoms [scoring above cut-off $(\geq 1)$ ] & $44(6.1)$ & 14 (12.7) & $30(4.9)$ & $10.0,0.002$ \\
\hline
\end{tabular}

Bold indicates statistical significance $(p<0.05)$

Common mental disorder (scoring above the cut-off on one or more screen measure for mental health), Mental health comorbidities (experiencing two or more common mental disorders concurrently)

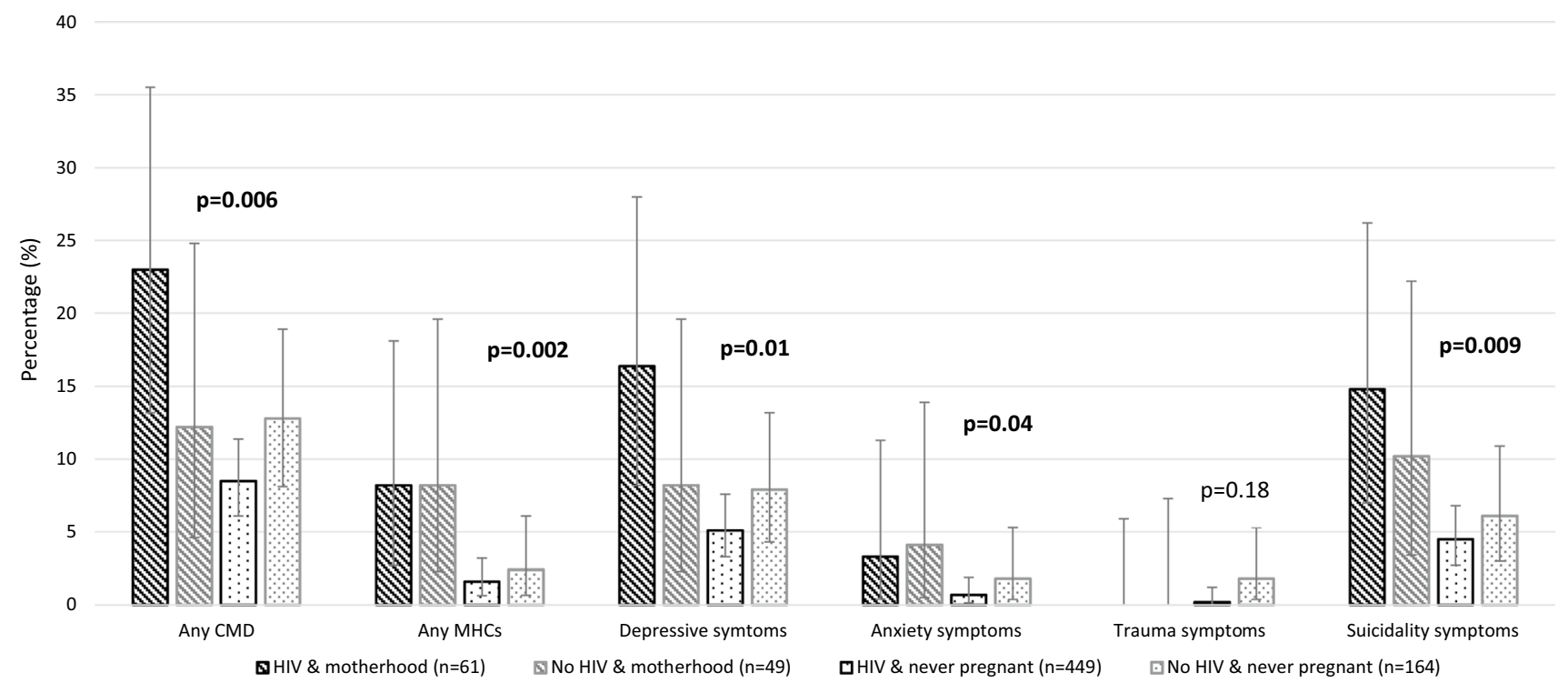

Fig. 2 Mental health outcomes stratified accordingly to combined motherhood and HIV status. CMD Common mental disorder (scoring above the cut-off on one or more screen measure for mental health),

\section{Prevalence of Common Mental Disorder According to the Syndemic of Adolescent Motherhood and HIV}

Figure 2 and Supplementary Table S1 presents the prevalence of probable common mental disorder disaggregated across four groups according to motherhood and HIV status. Prevalence of common mental disorder was found to be highest amongst adolescent mothers living with HIV (23.0\%). Likewise, prevalence of depressive symptomology was found to be highest among adolescent mothers living with HIV (16.4\%). Of note, the prevalence of probable mental health comorbidities was higher amongst mothers regardless of HIV status (adolescents living with HIV: 8.2\%, Adolescents not living with HIV: $8.2 \%$ ). Similar
MHCs Mental health comorbidities (experiencing two or more common mental disorders concurrently). Confidence intervals calculated at $95 \%$. $*$ One-sided $97.5 \%$ confidence interval

patterns were identified relating to anxiety and suicidality symptoms (see Fig. 2).

\section{Associations Between Combined Motherhood and HIV Status and Common Mental Disorder}

Table 3 and Fig. 3 presents logistic regression models exploring the cross-sectional associations between motherhood and HIV status and, common mental disorder. Within the multivariable models (model 1), the odds of experiencing probable mental health comorbidities were found to be elevated among those experiencing motherhood (regardless of HIV status), however, this did not reach significance (AOR: $2.64, p=0.08$; likely due to the small sample size experiencing mental health comorbidities). The association 


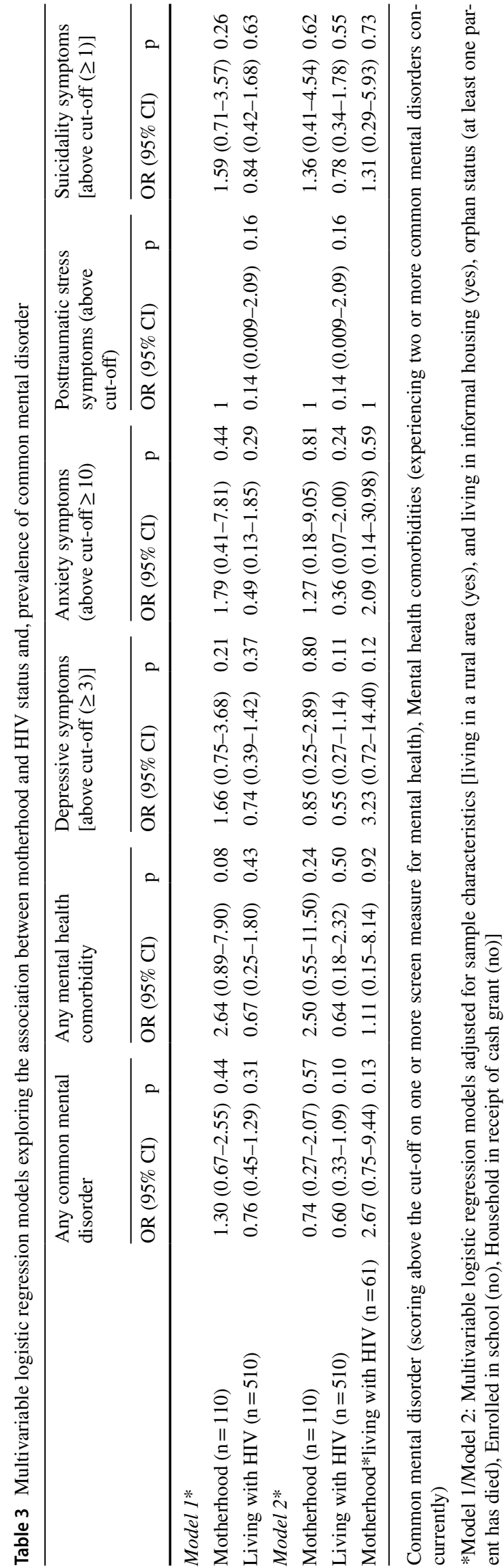

between motherhood and probable common mental disorder in the sample did not reach statistical significance (AOR: $1.30, p=0.44)$. Adjusted odds ratios suggest living with HIV was protective of probable common mental disorder/mental health comorbidities, however these associations did not reach statistical significance (model 1). Within the interaction models (model 2), a trend was identified for an exacerbating interaction effect between experiencing motherhood and living with HIV, predicting an increased likelihood of probable common mental disorder (AOR: 2.67, $\mathrm{p}=0.13$ ). A similar trend was identified for reported depressive symptomology (AOR: $3.23, p=0.12$ ), identifying depressive symptomology as a likely driver of reported probable common mental disorder within these analyses.

\section{Discussion}

This is the first known explicit exploration of mental health among adolescent mothers within sub-Saharan Africa with a focus on HIV status. Utilising data from a large prospective cohort study of adolescents living with HIV and a comparison group of adolescents not living with HIV within South Africa, this study explores the prevalence of probable common mental disorder according to experience of motherhood and, the syndemic of adolescent motherhood and HIV. The prevalence of adolescent motherhood was $15.2 \%$ (a figure in line with current national estimates) [21] and, over a tenth $(10.9 \%)$ were classified as reporting probable common mental disorder (either depression, anxiety, posttraumatic stress, or suicidality symptomology). These prevalence data indicate a need for attention to both adolescent mental health and particularly within the context of adolescent motherhood. The detailed exploration of associations in this study reveals three core findings. First, poor mental health was higher among mothers compared to adolescents who had never experienced pregnancy. Secondly, within univariate analyses prevalence of probable common mental disorder was found to be highest among adolescent mothers living with HIV (23.0\%) compared to those experiencing motherhood in the absence of a HIV diagnosis and non-mothers. Thirdly, prevalence of probable mental health comorbidities within unadjusted analyses (scoring above the cut-off on two or more measures of mental health symptomology) was found to be higher among adolescent mothers, regardless of HIV status, indicating that experience of pregnancy/motherhood may drive more complex mental health burden. This finding was supported within adjusted models, as a trend for adolescent mothers to be marginally more likely to report probable mental health comorbidities was identified. These findings highlight the commonality and potential complexity of mental health burden within the context of adolescent 


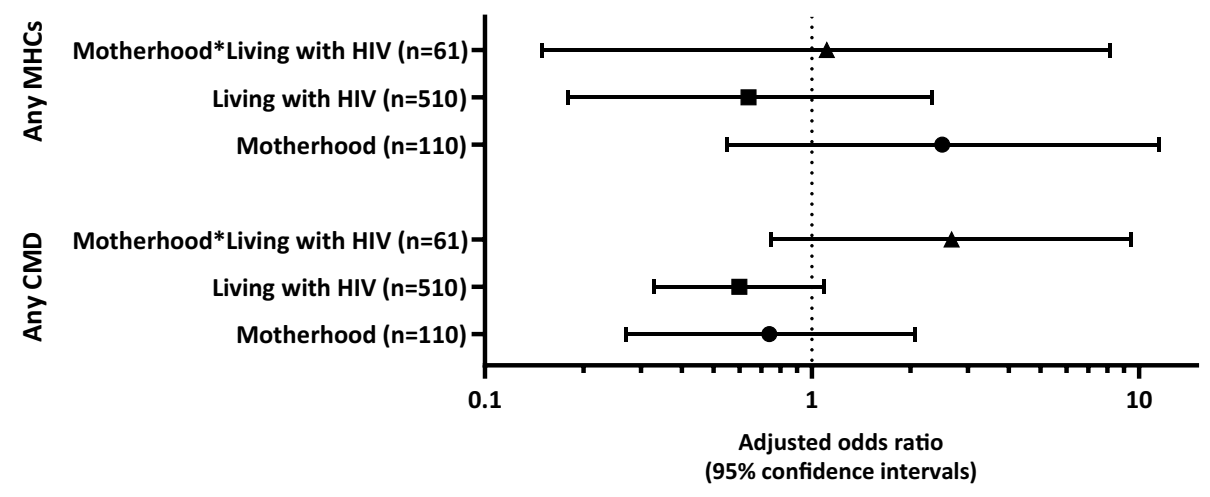

Fig. 3 Forest plot detailing adjusted odds ratios (with 95\% confidence intervals) according to combined adolescent pregnancy and HIV status. $C M D$ Common mental disorder (scoring above the cut-off on one or more screen measure for mental health), MHCs Mental health comorbidities (experiencing two or more common mental disorders

motherhood and HIV within South Africa and, the urgent need for further research and effective support for this group.

These findings extend literature identifying elevated prevalence of mental health burden among adolescent mothers $[23,24]$ and, highlight increased mental health burden (likely driven by probable depressive symptoms) among adolescents experiencing the dual impact of motherhood and living with HIV. As such, findings address a critical evidence gap as a recent systematic review identified no existing studies reporting the prevalence of common mental disorder among adolescent mothers living with HIV [37]. Adjusted models within analyses also highlight the potential complexity of probable common mental disorder among adolescent mothers within the context of HIV. While results suggest that adolescent motherhood may be associated with elevated poor mental health, adjusted models also indicate that living with HIV was potentially protective of mental health for this sample-a finding in contrast to previous literature. It should be noted that these associations did not reach statistical significance. Despite this, these findings, warrant further study to explore potential factors contributing to this pattern of results i.e. mode of HIV infection, time since HIV diagnosis, engagement with HIV services, resilience or access to social support may have implications for mental health within this population. Findings also highlight the experience of mental health comorbidities within adolescent motherhood, broadening previous investigations exploring singular mental health disorder [28]. The evidence base on mental health and pregnancy/motherhood is often limited to depressive symptomology with a reduced focus on anxiety, posttraumatic stress, and suicidal behaviours. In addition to highlighting elevated depressive symptomology among adolescents who have experienced motherhood in the sample, these data identify both anxiety and, worryingly, suicidality symptomology as prevalent among adolescent concurrently). Multivariable logistic regression models adjusted for sample characteristics [living in a rural area (yes), and living in informal housing (yes), orphan status (at least one parent has died), Enrolled in school (no), Household in receipt of cash grant (no)]

mothers. These data extend emerging literature identifying the commonality of suicidal ideation within pregnancy among HIV-affected populations in South Africa [72], providing evidence relating to adolescents. Furthermore, these data highlight the potential complexity of mental health experience (demonstrated through the presence of probable mental health comorbidities) for this group.

For adolescent mothers living with HIV, elevated common mental disorder is of particular concern as poor mental health has been found to impact HIV treatment uptake and adherence [73, 74], inclusive of PMTCT [75]—potentially having negative health consequences for both the adolescent and their child(ren). Mental health challenges have previously been found to be associated with parenting challenges i.e. harsh parenting practices [23] and sub-optimal child development outcomes [24, 28], bringing to light the importance of supporting adolescent maternal mental health for both the individual and their child(ren). A recent systematic review exploring psychosocial interventions for pregnant and parenting adolescents identified 17 interventions studies with small to moderate effects on positive mental health however, no studies were identified within low and middle income countries [76] — highlighting the need for tailored intervention among this group. There is a robust literature on effective interventions for maternal common mental disorder (mostly focused on depression) [77], inclusive of interventions for populations living with HIV within South Africa i.e. psycho-educational interventions [35, 78, 79]. Adapting such interventions for adolescents living with HIV may be of benefit. Future research may also be well placed to explore modifiable factors that contribute to common mental disorder among adolescent mothers living with HIV to better inform future programming. Possible factors driving elevated common mental disorder among this group may include stigma (related to both adolescent pregnancy/ 
motherhood and HIV), a lack of social support, poverty, parenting stress, knowledge, and timing of HIV diagnosis and, child HIV status [35, 80-82]. For the majority, contact with health services likely increases for these adolescents during the pregnancy and post-partum period. Inherently, this contact with antenatal and postnatal care services (as well as HIV care services for adolescents living with HIV), provides an opportunity to amalgamate differing branches of health service through providing screening, monitoring, and referral for common mental disorder.

These findings should be interpreted within the context of study limitations. Firstly, the data presented are crosssectional, as such the direction of causality cannot be established. Further, data is drawn from a sample of adolescents predominantly living with HIV (70.5\%), which not representative of national HIV prevalence estimates within South Africa [83]. Hence, this may have implications for the interpretation of results, as those living with HIV are over represented, thus limiting the generalisability of findings. Nevertheless, this study addresses a critical evidence gap relating to the exploration of mental health symptomology among in adolescent mothers living with HIV and provides a foundation for future studies exploring such topics. Secondly, the data presented are mostly self-report, including motherhood status and, mental health status. This remains common practice within the field of global mental health and was deemed the best available data given the setting of data collection and the scarcity of mental health/clinical services within the locality. Mental health symptoms above a cut-off are not diagnostic but give a clear indication of potential referral need and disorder. Thirdly, while these analyses use validated cut-offs on screening measures of mental health, these data do not examine the variability within the mental health experience (i.e. variation in screening scores). In recent years, there has been a call to shift away from binary classifications of mental disorder towards a continuum approach to mental health to better reflect the diversity and complexity of mental health experience [84, 85]. However, this is yet to be implemented within the global mental health field at scale [84]. Thus, to be able to establish need in relation to mental health in these early investigations of adolescent mothers living with HIV a binary classification system was utilised within analyses. While it is important not to detract from the contextual understanding of mental health experience or pathologise adolescents through such labelling, it is essential that poor mental health symptomology is identified to ensure that support can be provided to those in need. The classifications utilised within analyses were inclusive, both within the definition of common mental disorder utilised and the cut-offs used, to ensure that potential need was identified in this sample. While all measures have previously been used in populations in South Africa, further research is still required to confirm psychometric properties in relation shortened versions of some of the scales i.e. the CDI-S and Child PTSD checklist. Fourthly, given the distribution of participants within groups established from combined motherhood and HIV status, some of the regression models undertaken as part of these analyses may have been underpowered to detect associations. Nevertheless, trends within the models were identified and, the prevalence data within these analyses contribute to a critical gap within the evidence base relating to adolescent mothers living with HIV. Fifthly, these analyses focus solely on female adolescents. It was beyond the scope of this study to explore the mental health experience of adolescent fathers. As such, there remains an absence of literature regarding the experience of who have fathered a child [37]. Finally, it was beyond the scope of this study to explore pregnancy loss, parenting of multiple children and, some of the intricacies relating to the experience of mental health burden, adolescent motherhood, and HIV [i.e. how risk and protective factors contribute to such experiences, the impact of mode of maternal HIV infection and, the timing of common mental disorder symptomology (e.g. the post-partum period)]. However, the contribution of such factors should not be excluded from the discussion regarding these experiences. Future studies should be encouraged to further examine the experience of mental health for this group to allow for a greater understanding of modifiable mechanisms of effect for common mental disorder to better inform effective interventions among this group.

\section{Conclusions}

Mental health research, policy and provision is beginning to be given attention by international funding bodies and polygonal organisations however, resources, programming, and research capacity within the field remains low; limiting the production of evidence relevant to programming for adolescents within LMIC. For policy change to occur in relation to mental health, a core step is raising awareness of the burden of mental health challenges among the population and, identifying particularly vulnerable subgroups to allow for targeted responses [86]. This study identified a high prevalence of probable common mental disorder among a particularly vulnerable group and provides a foundation for further exploration regarding the needs of adolescent mothers within the context of HIV. This is the first explicit exploration of probable common mental disorder prevalence among adolescent mothers living with HIV within South Africa. Three core findings emerge: (1) results highlight elevated likely common mental disorder among adolescent mothers, (2) prevalence of probable common mental disorder was found to be greatest among those adolescents experiencing the syndemic of 
adolescent motherhood and HIV and, (3) likely mental health comorbidities were found to be prevalent among adolescent mothers regardless of HIV status. These data focus on syndemic conditions and highlight the potential need for integrated responses for adolescent mothers within South Africa (i.e. the amalgamation of antenatal, postnatal, and HIV care, with mental health screening).

Supplementary Information The online version contains supplementary material available at https://doi.org/10.1007/s10461-021-03474-8.

Acknowledgements We thank the adolescents and health-care facilities who participated in this study, the superb Mzantsi Wakho field teams and, the Oxford University and University of Cape Town support teams. This project was funded by the Nuffield Foundation [Grant No. CPF/41513], the UK Research and Innovation Global Challenges Research Fund award [Grant No. ES/S008101/1]; Evidence for HIV Prevention in Southern Africa, a UKAID programme managed by Mott MacDonald; Janssen Pharmaceutica NV; UNICEF Eastern and Southern Africa Office; and the International AIDS Society through the CIPHER grant (Grant No. 155-Hod; 2018/625-TOS). Further funding was provided by the Claude Leon Foundation (Grant No. 08 559/C) and Oak Foundation (Grant No. R46194/AA001). Additional funding was provided by the John Fell Fund (Grant Nos. 103/757 and 161/033); the University of Oxford's Economic and Social Research Council Impact Acceleration Account (Grant Nos. IAA-MT13-003; 1602-KEA-189; K1311-KEA-004); the Leverhulme Trust (Grant No. PLP-2014-095); Research England; the European Research Council (ERC) under the European Union's (EU) Seventh Framework Programme (Grant No. FP7/2007-2013)/ERC grant agreement 313421, the EU's Horizon 2020 research and innovation programme/ERC grant agreement 737476); the UK Medical Research Council (MRC) and the UK Department for International Development (DFID) under the MRC/DFID Concordat agreement, and by the Department of Health Social Care through its National Institutes of Health Research (MR/R022372/1I), and the Oxford University Clarendon-Green Templeton College Scholarship. KJR is supported by an Economic Social Research Council (ESRC) $\mathrm{PhD}$ studentship through the UBEL DTP. LS, LC, and ET receive funding from the UKRI GCRF Accelerating Achievement for Africa's Adolescents (Accelerate) Hub (Grant Ref: ES/S008101/1). ET receives additional support from the International AIDS Society through the CIPHER grant [2018/625-TOS] and the Fogarty International Center, National Institute on Mental Health, National Institutes of Health under Award Number K43TW011434. MB is supported by the National Health and Medical Research Council, Australia (Investigator Grant No. 1173043). Sponsors did not have any role within the design or conduct of the study or the analyses presented. The views expressed in written materials or publications do not necessarily reflect the official policies of funding organisations.

\section{Declarations}

Conflict of interest All authors declare no conflict of interest.

Ethical Approval Ethical approvals for the study were obtained from the Universities of Oxford (SSD/CUREC2/12-21) and Cape Town (CSSR 2013/14) as well as University College London for these analyses (14795/001). Additional local approvals were obtained from the South African Provincial Departments of Education and Health as well as the ethics committees of participating clinics.
Open Access This article is licensed under a Creative Commons Attribution 4.0 International License, which permits use, sharing, adaptation, distribution and reproduction in any medium or format, as long as you give appropriate credit to the original author(s) and the source, provide a link to the Creative Commons licence, and indicate if changes were made. The images or other third party material in this article are included in the article's Creative Commons licence, unless indicated otherwise in a credit line to the material. If material is not included in the article's Creative Commons licence and your intended use is not permitted by statutory regulation or exceeds the permitted use, you will need to obtain permission directly from the copyright holder. To view a copy of this licence, visit http://creativecommons.org/licenses/by/4.0/.

\section{References}

1. World Health Organization. Adoelscent development. Geneva: WHO; 2017.

2. Desa U. Revision of world population prospects. New York: United Nations Secretariat Department of Economic and Social Affairs; 2017.

3. Kessler RC, Angermeyer M, Anthony JC, et al. Lifetime prevalence and age-of-onset distributions of mental disorders in the world health organization's world mental health survey initiative. World Psychiatry. 2007;6(3):168.

4. Kim-Cohen J, Caspi A, Moffitt TE, Harrington H, Milne BJ, Poulton R. Prior juvenile diagnoses in adults with mental disorder: developmental follow-back of a prospective-longitudinal cohort. Arch Gen Psychiatry. 2003;60(7):709-17.

5. Copeland WE, Shanahan L, Costello EJ, Angold A. Childhood and adolescent psychiatric disorders as predictors of young adult disorders. Arch Gen Psychiatry. 2009;66(7):764-72.

6. Copeland WE, Adair CE, Smetanin P, et al. Diagnostic transitions from childhood to adolescence to early adulthood. J Child Psychol Psychiatry. 2013;54(7):791-9.

7. Eyre O, Thapar A. Common adolescent mental disorders: transition to adulthood. Lancet. 2014;383(9926):1366-8.

8. Copeland WE, Wolke D, Shanahan L, Costello EJ. Adult functional outcomes of common childhood psychiatric problems: a prospective, longitudinal study. JAMA Psychiat. 2015;72(9):892-9.

9. Prince M, Patel V, Saxena S, et al. No health without mental health. Lancet. 2007;370(9590):859-77.

10. Lund C, De Silva M, Plagerson S, et al. Poverty and mental disorders: breaking the cycle in low-income and middle-income countries. Lancet. 2011;378(9801):1502-14.

11. Gupta MD. The power of 18 billion: adolescents, youth, and the transformation of the future. UNFPA: The State of World Population; 2014.

12. Das J, Do Q-T, Friedman J, McKenzie D. Mental health patterns and consequences: results from survey data in five developing countries. World Bank Econ Rev. 2009;23(1):31-55.

13. Kieling $\mathrm{C}$, Baker-Henningham $\mathrm{H}$, Belfer $\mathrm{M}$, et al. Child and adolescent mental health worldwide: evidence for action. Lancet. 2011;378(9801):1515-25.

14. Shatkin JP, Belfer ML. The global absence of child and adolescent mental health policy. Child Adolesc Mental Health. 2004;9(3):104-8.

15. Patel V, Boyce N, Collins PY, Saxena S, Horton R. A renewed agenda for global mental health. Lancet (London, England). 2011;378(9801):1441.

16. World Health Organisation. Child and adolescent mental health. Geneva: WHO; 2019. 
17. Erskine H, Baxter A, Patton G, et al. The global coverage of prevalence data for mental disorders in children and adolescents. Epidemiol Psychiatr Sci. 2017;26(4):395-402.

18. Remschmidt H, Belfer M. Mental health care for children and adolescents worldwide: a review. World Psychiatry. 2005;4(3):147.

19. Belfer ML. Critical review of world policies for mental healthcare for children and adolescents. Curr Opin Psychiatry. 2007;20(4):349-52.

20. Patel V, Araya R, Chatterjee S, et al. Treatment and prevention of mental disorders in low-income and middle-income countries. Lancet. 2007;370(9591):991-1005.

21. Yah CS, Ndlovu S, Kutywayo A, Naidoo N, Mahuma T, Mullick $\mathrm{S}$. The prevalence of pregnancy among adolescent girls and young women across the Southern African development community economic hub: a systematic review and meta-analysis. Health Promot Perspect. 2020;10(4):325

22. Elfenbein DS, Felice ME. Adolescent pregnancy. Pediatr Clin. 2003;50(4):781-800.

23. Siegel RS, Brandon AR. Adolescents, pregnancy, and mental health. J Pediatr Adolesc Gynecol. 2014;27(3):138-50.

24. Dahmen B, Konrad K, Jahnen L, Herpertz-Dahlmann B, Firk C. Mental health of teenage mothers: impact on the next generation. Nervenarzt. 2019. https://doi.org/10.1007/s00115-018-0661-7.

25. O'Connor TG, Monk C, Burke AS. Maternal affective illness in the perinatal period and child development: findings on developmental timing, mechanisms, and intervention. Curr Psychiatry Rep. 2016;18(3):24.

26. Kingston D, Tough S. Prenatal and postnatal maternal mental health and school-age child development: a systematic review. Matern Child Health J. 2014;18(7):1728-41.

27. Ramchandani P, Psychogiou L. Paternal psychiatric disorders and children's psychosocial development. Lancet. 2009;374(9690):646-53.

28. Kvalevaag AL, Ramchandani PG, Hove O, Assmus J, EberhardGran M, Biringer E. Paternal mental health and socioemotional and behavioral development in their children. Pediatrics. 2013;131(2):e463-9.

29. Simbayi L, Zuma K, Zungu N, et al. South African national HIV prevalence, incidence, behaviour and communication survey, 2017: towards achieving the UNAIDS 90-90-90 targets. Cape Town: HSRC Press; 2019.

30 Dessauvagie AS, Jörns-Presentati A, Napp A-K, et al. The prevalence of mental health problems in sub-Saharan adolescents living with HIV: a systematic review. Global Mental Health. 2020. https://doi.org/10.1017/gmh.2020.18.

31. Fawzi MCS, Ng L, Kanyanganzi F, et al. Mental health and antiretroviral adherence among youth living with HIV in Rwanda. Pediatrics. 2016;138(4):e20153235.

32. Dow DE, Turner EL, Shayo AM, Mmbaga B, Cunningham CK, O'Donnell K. Evaluating mental health difficulties and associated outcomes among HIV-positive adolescents in Tanzania. AIDS Care. 2016;28(7):825-33.

33. Breuer E, Myer L, Struthers H, Joska JA. HIV/AIDS and mental health research in sub-Saharan Africa: a systematic review. Afr J AIDS Res. 2011;10(2):101-22.

34. Corcoran J. Teenage pregnancy and mental health. Societies. 2016;6(3):21.

35. Kapetanovic S, Dass-Brailsford P, Nora D, Talisman N. Mental health of HIV-seropositive women during pregnancy and postpartum period: a comprehensive literature review. AIDS Behav. 2014;18(6):1152-73.

36. Horwood C, Butler LM, Haskins L, Phakathi S, Rollins N. HIVinfected adolescent mothers and their infants: low coverage of HIV services and high risk of HIV transmission in KwaZuluNatal, South Africa. PLoS ONE. 2013;8(9):e74568.
37. Roberts KJ, Smith C, Cluver L, Toska E, Sherr L. Understanding mental health in the context of adolescent pregnancy and HIV in sub-Saharan Africa-a systematic review identifying a critical evidence gap. AIDS Behav. 2021. (In press).

38 Osok J, Kigamwa P, Stoep AV, Huang KY, Kumar M. Depression and its psychosocial risk factors in pregnant Kenyan adolescents: a cross-sectional study in a community health centre of Nairobi. BMC Psychiatry. 2018. https://doi.org/10.1186/ s12888-018-1706-y.

39. Kassebaum N, Kyu HH, Zoeckler L, et al. Child and adolescent health from 1990 to 2015: findings from the global burden of diseases, injuries, and risk factors 2015 study. JAMA Pediatr. 2017;171(6):573-92.

40. World Health Organisation. Adolescent mental health: fact sheet. Geneva: World Health Organisation (WHO); 2019.

41. Closson K, Dietrich JJ, Nkala B, et al. Prevalence, type, and correlates of trauma exposure among adolescent men and women in Soweto, South Africa: implications for HIV prevention. BMC Public Health. 2016;16(1):1191.

42. Bachmann S. Epidemiology of suicide and the psychiatric perspective. Int J Environ Res Public Health. 2018;15(7):1425.

43. Kovacs M, Staff M. Children's depression inventory (CDI): technical manual update. North Tonawanda: Multi-Health Systems; 2003.

44. Kovacs M. Children's depression inventory (CDI): technical manual update. Toronto: Multi-Health Systems; 1992.

45 Greenland S, Mansournia MA, Altman DG. Sparse data bias: a problem hiding in plain sight. BMJ. 2016. https://doi.org/10. 1136/bmj.i1981.

46. Kovacs M, Goldston D, Gatsonis C. Suicidal behaviors and childhood-onset depressive disorders: a longitudinal investigation. J Am Acad Child Adolesc Psychiatry. 1993;32(1):8-20.

47. Suliman S. Assessing post-traumatic responses among South African adolescents: a comparison of different methods. Cape Town: University of Cape Town; 2002.

48. Allgaier A-K, Frühe B, Pietsch K, Saravo B, Baethmann M, Schulte-Körne G. Is the children's depression inventory short version a valid screening tool in pediatric care? A comparison to its full-length version. J Psychosom Res. 2012;73(5):369-74.

49. Traube D, Dukay V, Kaaya S, Reyes H, Mellins C. Cross-cultural adaptation of the child depression inventory for use in Tanzania with children affected by HIV. Vulnerable Child Youth Stud. 2010;5(2):174-87.

50. Kim MH, Mazenga AC, Devandra A, et al. Prevalence of depression and validation of the beck depression inventory-II and the children's depression inventory-short amongst HIV-positive adolescents in Malawi. J Int AIDS Soc. 2014;17(1):18965.

51. Kagotho N, Patak-Pietrafesa M, Ssewamala FM, Kirkbride $\mathrm{G}$. Assessing the association between depression and savings for Kenyan youth using a validated child depression inventory measure. J Adolesc Health. 2018;62(1):S21-8.

52. Binagwaho A, Fawzi MCS, Agbonyitor M, et al. Validating the children's depression inventory in the context of Rwanda. BMC Pediatr. 2016;16(1):1-7.

53. Shenderovich Y, Boyes M, DegliEsposti M, et al. Relationships with caregivers and mental health outcomes among adolescents living with HIV: a prospective cohort study in South Africa. BMC Public Health. 2021;21(1):1-11.

54. Cluver L, Gardner F, Operario D. Psychological distress amongst AIDS-orphaned children in urban South Africa. J Child Psychol Psychiatry. 2007;48(8):755-63.

55. Sherr L, Croome N, Clucas C, Brown E. Differential effects of single and double parental death on child emotional functioning and daily life in South Africa. Child Welfare. 2014;93(1):149.

56. Woollett N, Cluver L, Bandeira M, Brahmbhatt H. Identifying risks for mental health problems in HIV positive adolescents 
accessing HIV treatment in Johannesburg. J Child Adolesc Mental Health. 2017;29(1):11-26.

57. Reynolds CR, Richmond BO. What I think and feel: a revised measure of children's manifest anxiety. J Abnorm Child Psychol. 1978;6(2):271-80.

58. Gerard AB, Reynolds CR. Characteristics and applications of the revised children's manifest anxiety scale (RCMAS). In: Maruish ME, editor. The use of psychological testing for treatment planning and outcomes assessment. Mahwah: Lawrence Erlbaum Associates Publishers; 1999. p. 323-40.

59. Boyes ME, Cluver LD. Performance of the revised children's manifest anxiety scale in a sample of children and adolescents from poor urban communities in Cape Town. Eur J Psychol Assess. 2013;29(2):113-20.

60. Amaya-Jackson L, McCarthy G, Cherney M, Newman E. Child PTSD checklist. Durham: Duke University Medical Center; 1995.

61. Boyes ME, Cluver LD, Gardner F. Psychometric properties of the child PTSD checklist in a community sample of South African children and adolescents. PLoS ONE. 2012;7(10):e46905.

62. Newman E, Amaya-Jackson L. Assessment of trauma instruments for children. In: Paper presented at 12th international conference for traumatic stress studies. San Francisco, USA; 1996.

63 American Psychiatric Association. Diagnostic and statistical manual of mental disorders DSM-5. Washington: American Psychiatric Association; 2013.

64. Seedat S, Nyamai C, Njenga F, Vythilingum B, Stein DJ. Trauma exposure and post-traumatic stress symptoms in urban African schools: survey in Cape Town and Nairobi. Br J Psychiatry. 2004;184(2):169-75.

65. Seedat S, Van Nood E, Vythilingum B, Stein DJ, Kamlner D. School survey of exposure to violence and posttraumatic stress symptoms in adolescents. South Afr J Child Adolesc Ment Health. 2000;12(1):38-44.

66. Sheehan DV, Sheehan KH, Shytle RD, et al. Reliability and validity of the mini international neuropsychiatric interview for children and adolescents (MINI-KID). J Clin Psychiatry. 2010;71(3):313-26.

67 Lecrubier Y, Sheehan DV, Weiller E, et al. The mini international neuropsychiatric interview (MINI). A short diagnostic structured interview: reliability and validity according to the CIDI. Eur Psychiatry. 1997;12(5):224-31.

68. Sheehan D, Lecrubier Y, Sheehan KH, et al. The validity of the mini international neuropsychiatric interview (MINI) according to the SCID-P and its reliability. Eur Psychiatry. 1997;12(5):232-41.

69. Stata Statistical Software. Release 15 [computer program]. Colllege Station: StataCorp LLC; 2017.

70. Mickey RM, Greenland S. The impact of confounder selection criteria on effect estimation. Am J Epidemiol. 1989;129(1):125-37.

71. Maldonado G, Greenland S. Simulation study of confounderselection strategies. Am J Epidemiol. 1993;138(11):923-36.

72 Rochat TJ, Bland RM, Tomlinson M, Stein A. Suicide ideation, depression and HIV among pregnant women in rural South Africa. Health. 2013. https://doi.org/10.4236/health.2013.53A086.
73. Adejumo OA, Malee KM, Ryscavage P, Hunter SJ, Taiwo BO. Contemporary issues on the epidemiology and antiretroviral adherence of HIV-infected adolescents in sub-Saharan Africa: a narrative review. J Int AIDS Soc. 2015;18(1):20049.

74. Taddeo D, Egedy M, Frappier J-Y. Adherence to treatment in adolescents. Paediatr Child Health. 2008;13(1):19-24.

75. Psaros C, Smit JA, Mosery N, et al. PMTCT adherence in pregnant South African women: the role of depression, social support, stigma, and structural barriers to care. Ann Behav Med. 2020;54(9):626-36.

76. Laurenzi CA, Gordon S, Abrahams N, et al. Psychosocial interventions targeting mental health in pregnant adolescents and adolescent parents: a systematic review. Reprod Health. 2020;17:1-15.

77. Alderdice F, McNeill J, Lynn F. A systematic review of systematic reviews of interventions to improve maternal mental health and well-being. Midwifery. 2013;29(4):389-99.

78. Futterman D, Shea J, Besser M, et al. Mamekhaya: a pilot study combining a cognitive-behavioral intervention and mentor mothers with PMTCT services in South Africa. AIDS Care. 2010;22(9):1093-100.

79. Mundell JP, Visser MJ, Makin JD, et al. The impact of structured support groups for pregnant South African women recently diagnosed HIV positive. Women Health. 2011;51(6):546-65.

80. Hill LM, Maman S, Groves AK, Moodley D. Social support among HIV-positive and HIV-negative adolescents in Umlazi, South Africa: changes in family and partner relationships during pregnancy and the postpartum period. BMC Pregnancy Childbirth. 2015;15(1):1-9.

81. Toska E, Laurenzi CA, Roberts KJ, Cluver L, Sherr L. Adolescent mothers affected by HIV and their children: a scoping review of evidence and experiences from sub-Saharan Africa. Glob Public Health. 2020;15(11):1655-73.

82. Boyes ME, Cluver LD, Meinck F, Casale M, Newnham E. Mental health in South African adolescents living with HIV: correlates of internalising and externalising symptoms. AIDS Care. 2019;31(1):95-104

83. UNICEF. HIV/AIDS Global and regional trends [Internet]. New York, USA; 2020 [cited 2021 Sept 1]. Available from: https://data. unicef.org/topic/hivaids/global-regional-trends/.

84. Patel V, Saxena S, Lund C, et al. The lancet commission on global mental health and sustainable development. Lancet. 2018;392(10157):1553-98.

85. Jacob KS, Patel V. Classification of mental disorders: a global mental health perspective. Lancet. 2014;383(9926):1433-5.

86. Laurenzi CA, Skeen S, Gordon S, et al. Preventing mental health conditions in adolescents living with HIV: an urgent need for evidence. J Int AIDS Soc. 2020;23:e25556.

Publisher's Note Springer Nature remains neutral with regard to jurisdictional claims in published maps and institutional affiliations.

\section{Authors and Affiliations}

\section{Kathryn J. Roberts ${ }^{1}\left(\mathbb{0} \cdot\right.$ Colette Smith $^{1} \cdot{\text { Lucie } \text { Cluver }^{2,3} \cdot \text { Elona Toska }^{2,4,5} \cdot \text { Siyanai Zhou }}^{4} \cdot$ Mark Boyes $^{6}$. Lorraine Sherr ${ }^{1}$}

Kathryn J. Roberts

k.roberts@ucl.ac.uk
Institute for Global Health, University College London, London, UK 
2 Department of Social Policy and Intervention, University of Oxford, Oxford, UK

3 Department of Psychiatry and Mental Health, University of Cape Town, Cape Town, South Africa

4 Centre for Social Science Research, University of Cape Town, Cape Town, South Africa
5 Department of Sociology, University of Cape Town, Cape Town, South Africa

6 Curtin enAble Institute, Faculty of Health Sciences, Curtin University, Bentley, Perth, Australia 